\title{
The Utility of Routine Endoscopic Distal Duodenal Biopsy in Patients with Irritable Bowel Syndrome for Celiac Disease Diagnosis; First Study from Nepal
}

\author{
Mohan Khadka* \\ Institute of Medicine, Tribhuvan University Teaching Hospital, Kathmandu, Nepal \\ *Corresponding author: khamohan620@gmail.com
}

\begin{abstract}
Aim: To investigate the utility of routine endoscopic distal duodenal biopsy in patients with Irritable Bowel Syndrome (IBS) for the diagnosis of Celiac Disease (CD). Method: This study was conducted at the outpatient Department of Gastroenterology at the Institute of Medicine, Tribhuvan University, Kathmandu, Nepal. One hundred patients with IBS selected by Rome III Criteria were prospectively evaluated from April 2015 through March 2016. Esophagogastroduodenoscopy (EGD) was performed on all patients, with four-quadrant distal duodenal biopsies obtained from each patient. The biopsy sections were studied for Marsh-type histology, a histology characteristic of CD. Patients with Marsh-type histology had Immunoglobulin-A anti-tissue transglutaminase (IgA anti tTG) serology ordered, were recommended to start a gluten-free diet (GFD), and were followed up in clinic in 3-6 weeks. The prevalence of CD (defined as Marsh histology grade I-III with serology positive responding to GFD) was calculated in patients with IBS as a whole and among IBS subtypes. Statistical analysis was done using SPSS version 19 software (Chicago, IL, USA). Results: Of the 100 IBS patients evaluated, 50 patients had abnormal duodenal histology. Among these 50 patients, 13 had Marsh-type histology, and the remaining 37 had non-specific duodenitis. Of the 13 patients with Marsh-type histology, 4 were seropositive for CD and 9 were seronegative. The overall prevalence of $\mathrm{CD}$ was $4 \%$. All 4 seropositive and 2 out of 9 seronegative patients responded to GFD in 3 to 6 weeks. Conclusion: Routine endoscopic distal duodenal biopsy is useful for the diagnosis of CD in patients with IBS as it could also detect the disease in patients with negative celiac serology.
\end{abstract}

Keywords: irritable bowel syndrome, celiac disease, $H L A-D Q 2 / D Q 8$, marsh classification, gluten free diet

Cite This Article: Mohan Khadka, "The Utility of Routine Endoscopic Distal Duodenal Biopsy in Patients with Irritable Bowel Syndrome for Celiac Disease Diagnosis; First Study from Nepal." International Journal of Celiac Disease, vol. 6, no. 1 (2018): 14-19. doi: 10.12691/ijcd-6-1-4.

\section{Introduction}

Irritable bowel syndrome (IBS), as defined by the Rome III criteria, is recurrent abdominal pain or discomfort at least 3 days per month in the last 3 months which is associated with two or more of the followings: improvement with defecation; onset associated with a change in frequency of stool and onset associated with a change in form or appearance of stool and the criteria are fulfilled with symptoms onset 6 months prior to diagnosis [1]. IBS is a common functional gastrointestinal disorder and is estimated to affect between $9 \%$ and $18 \%$ of the general population [2]. Besides the morbidity caused by IBS, it creates an economic burden to society through increased sick leave and consumption of healthcare resources $[3,4]$. Numerous guidelines have proposed that additional diagnostic tests are not necessary in patients with typical IBS symptoms in absence of red flags suggestive of organic disease such as blood in stools, nocturnal symptoms, unintentional weight loss, anorexia because the probability of having an organic pathology is very low [2,5]. It is generally accepted that IBS is a multifactorial condition, and patients having this disease are actually a heterogeneous group with different underlying mechanisms which are so far unknown [6].

For more than two decades, there is growing interest in the myriad of clinical presentations of Celiac Disease (CD), including IBS symptoms. CD can initially manifest as symptoms of IBS, predominantly in the form of diarrhea [7]. The classic presentation of $\mathrm{CD}$ as a malabsorption syndrome, also known as the "iceberg" condition, has become less common [8]. The variety of clinical presentations often results in a delay in diagnosis, which may result in serious complications [9]. A number of studies have suggested that carrying out $\mathrm{CD}$ screening tests in patients with IBS symptoms could be a cost-effective and beneficial strategy, given the higher prevalence of $\mathrm{CD}$ in subjects with IBS than in the general population [10-16]. In a meta-analysis of 14 case-controlled studies [17] in which 2278 individuals diagnosed with IBS were analyzed; the prevalence of IgA anti tTG and/or anti-endomysial antibodies (anti-EMA) was $2.94 \%$ (95\% CI $1.36-6.35)$ whereas the prevalence of histological demonstrated CD was $4.1 \%(1.9-7.0)$. Furthermore, two decision-analytic 
models have evaluated the cost-effectiveness of CD evaluation with serology followed by histology in seropositive cases and have concluded that it is beneficial if the prevalence of $\mathrm{CD}$ in the general population is greater than $1 \%$ [18]. Based on this evidence, the American College of Gastroenterology recommends routine serologic screening for $\mathrm{CD}$ in subjects with IBS with diarrhea (IBS-D) and mixed IBS (IBS-M) (Grade 1B Recommendation) [19]. However, in a study in Iran, sensitivity of serum IgA anti-tTG for serologic diagnosis of CD was found to be only $36 \%$, underestimating the true prevalence of CD [20]. Although the diagnosis of $\mathrm{CD}$ may be suspected on clinical grounds or as a result of abnormal serologic tests, biopsy of the small intestine has remained the gold standard test to establish the diagnosis [21].

In another study, routine distal duodenal biopsy in consecutive 262 patients with IBS symptoms revealed microscopic abnormalities including marsh type histology in $80 \%$, out of which $5 \%$ were definitely diagnosed as CD by further serologic test [22]. In a 30 year prospective study from 1982 to 2011 at a university teaching hospital in Canada, 9,665 patients with different gastrointestinal complaints underwent endoscopy with duodenal biopsies, of which 234 (2.4\%) exhibited changes of Celiac Disease (Marsh class II to III) [23].

Nevertheless, recent studies have shown results contradicting these findings. In a four-center study in the United States conducted on patients with non-constipated IBS, Cash et al. [24] found that the prevalence of CD was similar to that of a control population $(0.41 \%$ vs. $0.44 \%$, $\mathrm{p}=0.99$ ). In another study by Choung et al. [25] revealed that seropositive $\mathrm{CD}$ prevalence in subjects with symptoms suggestive of functional gastrointestinal disorders was only $1 \%$.

There is neither a published study about prevalence of $\mathrm{CD}$ in the general population nor in patients with IBS in Nepal. Though screening for CD by serologic testing is common practice worldwide, it might be cost effective to screen $\mathrm{CD}$ by performing routine endoscopic distal duodenal biopsy, which is seen as the gold standard for diagnosis too. The aim of this study was to investigate the utility of routine endoscopic distal duodenal biopsy in patients with IBS for the diagnosis of CD.

\section{Materials and Methods}

Ethical approval for the study was obtained from the Institutional Review Board at our institution. The potential implication of a positive result for CD was explained to all participants, and their written consent was obtained. Patients that sought medical attention for gastrointestinal symptoms suggestive of IBS in our department within the time frame of April 2, 2015 and March 30, 2016 were prospectively evaluated. Subjects that met the Rome III Criteria for IBS were included in the study [1]. Clinical evaluation included background, a standard symptom questionnaire for assessment of ROME III criteria, physical examination, and baseline laboratory investigations including complete blood count, metabolic panel, liver, renal, and thyroid function test, random blood sugar, erythrocyte sedimentation rate, and fecal occult blood test.
All patients received ultrasound of the abdomen and pelvis. Patients over age 50 or family history of colorectal carcinoma or inflammatory bowel disease also received colonoscopy.

After overnight fasting, esophagogastroduodenoscopy (EGD) was performed in all patients. During the EGD, gross distal duodenal findings were noted, and four quadrant biopsies were obtained from the second and third parts of the duodenum. Biopsies were fixed in $4 \%$ buffered paraformaldehyde overnight and further processed and examined by a pathologist as per the protocols of our pathology department. The stained sections were examined for the possible occurrence of CD using modified Marsh's classification. The classification for grading of severity of histopathologic changes is shown in [Table 1]. If histologic diagnosis was compatible with $\mathrm{CD}$, then $\operatorname{IgA}$ anti tTG serology was ordered. Five $\mathrm{mL}$ of blood was drawn from all the subjects with Marsh I and above. The serum was separated to perform the IgA anti tTG through the serum enzyme immunoassay by Super Religare Laboratories (SRL) Reference Lab, Kathmandu, Nepal. Titers under $12 \mathrm{U} / \mathrm{ml}$ were considered negative, 12-18 $\mathrm{U} / \mathrm{ml}$ were equivocal, and more than $18 \mathrm{U} / \mathrm{ml}$ were positive and considered as seropositive for CD. Patients with suspected $C D$ by the presence of Marsh-type abnormal histology, irrespective of celiac serology status, were recommended to follow a gluten free diet (GFD) and received nutrition counseling through our institution. These patients were followed up in clinic 3-6 weeks after GFD to monitor clinical progress.

Descriptive statistics were carried out and CD prevalence was calculated in patients with IBS. Prevalence was compared through the chi-squared test among different IBS subtypes. Differences among IBS subtypes were calculated using the Kruskal Wallis test. Differences of prevalences between seronegative and seropositive CD were calculated with regard to gender distribution by Pearson Chi-square test. A P-value of less than 0.05 was considered statistically significant. Microsoft word and Excel were used to generate tables and graphs. The analysis was done with SPSS version 19 software (Chicago, IL, USA).

\section{Results}

A total of 100 subjects with IBS were evaluated. Patients were from various regions of Nepal. There were 59 male and 41 female subjects with an age range of 17 to 64 years, with mean age 33.8 (standard deviation 12) and median age 30.5. As seen in Graph 1, the majority of study participants were young adults (42\%), with few patients above age $60(4 \%)$.

Out of 100 IBS patients, 59 patients had irritable bowel syndrome-diarrhea predominant (IBS-D), 21 had irritable bowel syndrome-constipation predominant (IBS-C), 11 had irritable bowel syndrome-unsubtyped (IBS-U) and 9 had irritable bowel syndrome-mixed (IBS-M) as shown in Graph 2. The duration of IBS symptoms ranged from 6 months to 10 years, with median of 12 months. Average time from appearance of clinical symptoms to the definitive diagnosis of $\mathrm{CD}$ was 41 months. 


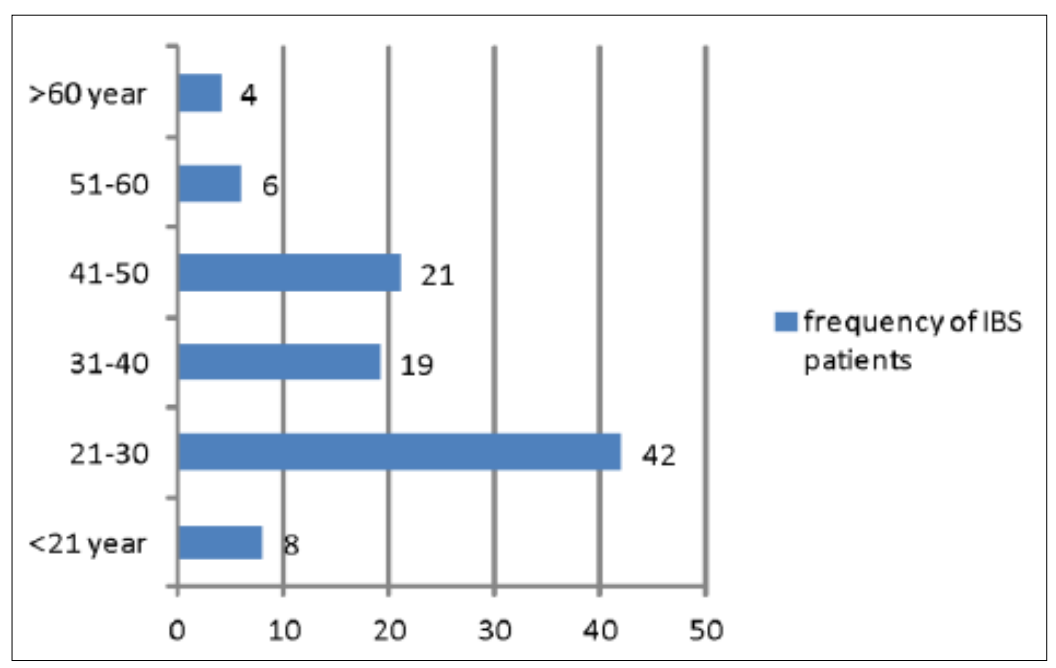

Graph 1. Age category distribution of IBS patients

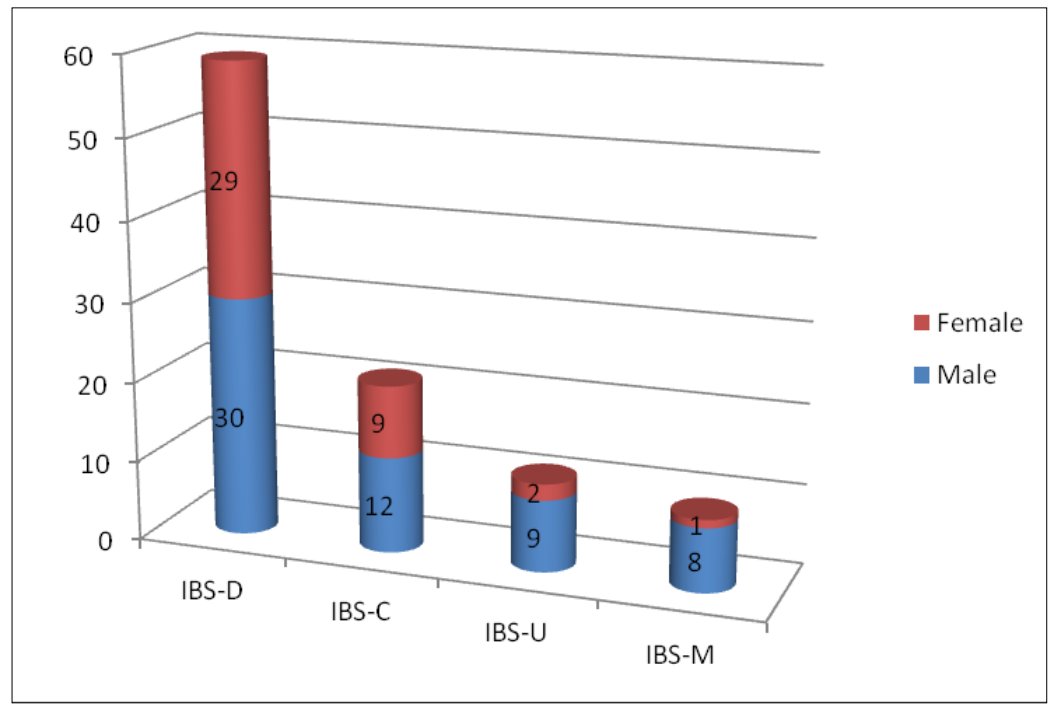

Graph 2. IBS subtypes distribution with gender

\subsection{EGD: Distal duodenum Gross and Histopathology}

The second part of the duodenum was found to be macroscopically normal in 90 patients and abnormal only in 10 patients. Of the 10 patients with macroscopic abnormality, mosaic pattern was seen in 2 , scalloping pattern in 1 , mucosal nodularity in 1, and erythematous duodenitis in 6 . Histopathologic examination of the distal duodenal biopsies revealed abnormal histology in 50 patients, out of which nonspecific duodenitis was seen in 37 , and modified Marsh type abnormal histology (Table 1) was found in 13. Among 13 patients with modified Marsh grading, Marsh I was found in 8, Marsh II in 4, and Marsh IIIa in 1 (Table 2).

Table 1. The Modified Marsh Classification of Celiac Disease

\begin{tabular}{|c|c|c|c|}
\hline Type & $\begin{array}{c}\text { Intraepithelial lymphocytes } \\
\text { per 100 duodenal enterocytes }\end{array}$ & Crypts \\
\hline $\mathbf{0}$ & $<30$ & Normal & Normal \\
\hline $\mathbf{1}$ & $>30$ & Increased & Normal \\
\hline $\mathbf{2}$ & $>30$ & Increased & Increased \\
\hline 3a & $>30$ & Increased & Marked atrophy \\
\hline 3b & $>30$ & Absent \\
\hline 3c & & atrophy \\
\hline
\end{tabular}

Table 2. Endoscopic gross and microscopic findings

\begin{tabular}{|c|c|c|c|c|c|c|c|}
\hline \multirow{2}{*}{\multicolumn{2}{|c|}{ Gross }} & \multicolumn{6}{|c|}{ Histopathology } \\
\hline & & \multicolumn{5}{|c|}{ Abnormal } & Normal \\
\hline \multirow[t]{2}{*}{ Abnormal } & \multirow[t]{2}{*}{ Normal } & \multicolumn{4}{|c|}{ Marsh type } & \multirow[t]{2}{*}{ Non-specific chronic duodenitis } & \\
\hline & & $\mathrm{I}$ & II & IIIa & Total & & \\
\hline 10 & 90 & 8 & 4 & 1 & 13 & 37 & 50 \\
\hline
\end{tabular}




\subsection{Serologic Testing and Diagnosis of CD}

Serologic testing for IgA anti tTG was performed in all 13 patients with Marsh type abnormal histology. IgA anti tTG was significantly higher than the upper limit of normal in 4 patients, and thus was labeled as seropositive Celiac Disease (SPCD). Among SPCD patients, 3 were male and 1 was female. The remaining 9 patients with Marsh-type abnormal histology had normal serum IgA anti tTG and hence were labeled as probable Celiac Disease with seronegativity (SNCD). Among SNCD patients, 6 were male and 3 were female. This difference in gender distribution was not statistically significant (Pearson Chi-square $=0.90, \mathrm{P}=0.764$ ). Among the $4 \mathrm{SPCD}$ patients, 3 belonged to the IBS-D category and 1 belonged to the IBS-C category; these patients were $24,23,24,43$ years old, respectively. Serum IgA level was normal in all SNCD patients, ruling out the possibility of selective IgA deficiency. As shown in Table 3, the prevalence of $\mathrm{CD}$ in IBS-D was 3 out of $59(5.1 \%)$ and in IBS-C was 1 in 21 $(4.8 \%)$ and there was no seropositive CD patient with IBS-U or IBS-M. Chi square test (Kruskal Wallis test) showed there was no statistically significant difference of prevalence of SPCD among different subgroups of IBS (Chi-square $=1.035$, $\mathrm{p}$ value $=0.596$ ).

On follow in 3 to 6 weeks on a gluten free diet, all 4 patients with SPCD had improvement in their IBS symptoms where as only 2 out of the 9 SNCD patients showed symptoms improvement (Graph 3). This supports that Marsh-type histology is not specific for Celiac Disease. The prevalence of $\mathrm{CD}$ in our study population of patients with IBS was likely $6 \%$, with 4 SPCD patients and 2 SNCD patients who showed symptoms improvement with a gluten free diet.

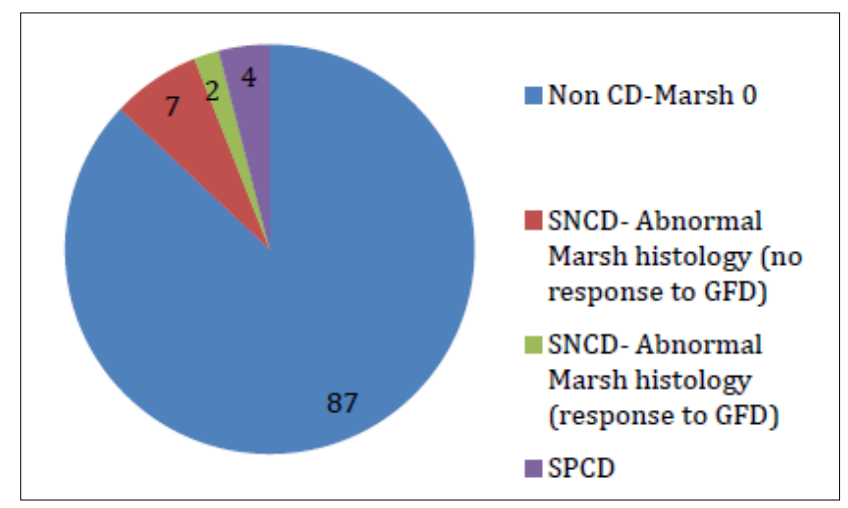

Graph 3. Distribution of SPCD, SNCD and Non CD

Table 3. Distribution of Celiac Disease (histology suggestive) among subtypes of IBS

\begin{tabular}{ccccccc}
\hline IBS (total=100) & \multicolumn{5}{c}{ Celiac Disease (total=13) } \\
& \multicolumn{7}{c}{ SNCD } & SPCD & \\
& M & F & Total & M & F & Total \\
\hline IBS-D (59) & 3 & 2 & 5 & 2 & 1 & 3 \\
IBS-C (21) & 1 & 1 & 2 & 1 & 0 & 1 \\
IBS-U (11) & 1 & & 1 & 0 & 0 & 0 \\
IBS-M (9) & 1 & & 1 & 0 & 0 & 0 \\
CD & 6 & 3 & 9 & 3 & 1 & 4 \\
\hline
\end{tabular}

\section{Discussion}

According to our results, the prevalence of definitive $\mathrm{CD}$ in our cohort of IBS patients as diagnosed by Marsh type histology, positive serology and response to a gluten free diet was $4 \%$. This parallels the prevalence of $C D$ in IBS patients found in the previous meta-analyses and studies reported in Iran [6], United Kingdom [10,13], Sweden [12], Turkey [15], Germany [27], Italy [28] and Latvia [29].

Therefore, evaluation for $\mathrm{CD}$ in patients that have prolonged IBS symptoms, especially those who present with the diarrhea subtype, appears to be justified in our population as well. Although the most recent studies in the United States question the justification of searching for CD $[24,25]$ in patients with IBS, our study supported this. A limitation of our study is that we did not have a control group. However, for comparison purposes, we reviewed a few studies showing prevalences of $\mathrm{CD}$ in the general population as well as in high risk groups from India. These populations are comparable with Nepal's population in terms of food habits, ethnicity and geographical proximity. In one study, the serologic testing of 198 symptomatic patients out of 4,347 total school children in Punjab, North India, yielded a seropositive $\mathrm{CD}$ prevalence of at least 1 in $310(0.3 \%)$ in the overall sample [30]. During recent years, it has been shown that in wheat consuming states such as Punjab, Uttar Pradesh, Delhi, Bihar, the prevalence of CD is higher (1\%) than the prevalence in non-wheat-consuming states like Kerala [31]. Studies on prevalence of CD in Uttar Pradesh as well as from Delhi have shown similarity to the European susceptibility patterns $[26,32,33]$. However, past studies indicate that there is a difference in the prevalence of $\mathrm{CD}$ between the northern and southern part of India [34,35].

Serological testing with $\operatorname{IgA}$ anti tTG have a positive predictive value of $75 \%-80 \%$. However, sero-negative CD is well-recognized in milder degrees of villous atrophy specifically Marsh I [36]. Marsh I type histologic changes may be seen in other conditions such as Helicobacter pylori infection, Cow milk allergy, small intestinal bacterial overgrowth, non-steroidal anti-inflammatory drug induced enteropathy, tropical sprue, and inflammatory bowel disease. In our study, the sero-negative histology positive probable CD was $9 \%$ of test subjects, out of which few could have been true $\mathrm{CD}$ as two of the nine patients showed improvement of symptoms with gluten free diet. According to the study [36], there is a possibility of sero-negative CD especially in Marsh type I, which could increase the true prevalence of $\mathrm{CD}$ even higher than the $4 \%$ seen in our study.

It is our opinion that IBS patients who present with prolonged or difficult to control symptoms should be tested for CD. In the meta-analysis by Ford [16], the odds ratio for detecting $\mathrm{CD}$ in patients with IBS through IgA anti EMA or tTG testing was $1.97(95 \%$ CI $0.60-6.43)$ at the primary care level, but it was almost double i.e. 3.99 (1.04-15) when studies were conducted at referral centers. Once the guidelines for primary care IBS management begin to recommend serologic screening for $\mathrm{CD}$ in patients meeting the criteria for IBS, the yield of testing in secondary and tertiary care centers may start to decline. 
The majority of the previous studies have included patients that were diagnosed with IBS based on clinical criteria or the Rome II criteria [16,24,25]. Some studies did not evaluate patients according to IBS subtypes. The prevalence of $C D$ is significantly higher in IBS-D and IBS-M as shown by many studies and also advocated by the American College of Gastroenterology in 2009 [19], however our study showed higher prevalence in IBS-C $(4.5 \%)$ in addition to IBS-D (5\%). Our findings of prevalences were even lower than a recent study that showed $14 \%$ in IBS-D and $8 \%$ in IBS-C [37]. Contrary to our study outcome, another prospective observational study [38] with 742 patients with presumed IBS showed the prevalence of CD in patients with IBS-D $(6.80 \%, 95 \%$ CI: 3.36, 10.23), that was significantly higher than in patients with IBS-C $(1.68 \%, 95 \% \mathrm{CI}: 0.35,3.01)$. The prevalence of CD found in our study in IBS patients according to the Rome III criteria was similar to that reported in more recent studies using the same criteria. Examples of this include a study by Patel et al. in Canada that reported a prevalence of $2.9 \%$ [39], or the Iranian study by Bakhshipour A, in which prevalence was 5.5\% [40].

In a prospective study of 262 patients who underwent routine duodenal biopsy, abnormal histology was revealed in $81 \%$, out of which $6 \%$ were compatible with Marsh type abnormal histology, and the remaining had nonspecific duodenitis [22]. In our study, it is important to mention that $50 \%$ of our biopsied cases $(n=100)$ had abnormal histology out of which 13 patients (13\%) had Marsh type abnormal histology.

A limitation of our study is that we did not evaluate for the possibility of non-celiac gluten sensitivity (NCGS) in the patients for IBS symptoms. In patients meeting the Rome III criteria for IBS, NCGS has been diagnosed in a high percentage of cases, and they have been sub-grouped as having gluten sensitive IBS [41,42]. Another limitation of our study was that we did not perform the DQ2/DQ8 haplotypes of the major histocompatibility complex [19]. It could be helpful in Marsh I-II patients with negative celiac serology, such as in our 9 patients to effectively rule out CD.

\section{Conclusion}

We conclude that the routine endoscopic distal duodenal biopsy in patients with irritable bowel syndrome for the diagnosis of Celiac Disease is useful as it could also detect the disease in patients with negative celiac serology.

We hope this probably first preliminary study from Nepal encourages other researchers to conduct more systematically designed, randomized controlled studies with a larger sample size in order to draw definitive conclusion.

\section{Conflicts of Interest}

The author declares that there is no conflict of interest.

\section{Acknowledgements}

I would like to thank Dr. Elizabeth Snyder for her wonderful contribution in editing technical parts, Dr. Bed
Chhatkuli, Dr. Santosh Sapkota for sincere support from USA and whole Gastroenterology and Pathology team of Institute of Medicine, Tribhuvan University Teaching hospital, Nepal for facilitating the research.

\section{References}

[1] Drossman DA. The functional gastrointestinal disorders and the Rome III process. Gastroenterology 2006; 130: 1377-9.

[2] Valenzuela J, Alvarado J, Cohen H, Damiao A, Francisconi C, Frugone L, Gonzalez JC, Hernandez A et al. Latin American consensus document on irritable bowel syndrome. Gastroenterol Hepatol 2004; 27: 325-43

[3] Everhart JE and Renault PF: Irritable bowel syndrome in office-based practice in the United States. Gastroenterology 100: 998-1005, 1991.

[4] Harvey RF, Salih SY and Read AE: Organic and functional disorders in 2000 gastroenterology outpatients. Lancet 1: 632-634, 1983.

[5] Cash BD, Schoenfeld P, Chey WD. The utility of diagnostic test in irritable bowel syndrome patients: a systematic review. Am J Gastroenterol 2002; 97: 2812-9.

[6] Shahbazkhani B, Forootan M, Merat S, Akbari MR, Nasserimoghadam S, Vahedi H, Malekzadeh R. Coeliac disease presenting with symptoms of irritable bowel syndrome. Aliment Pharmacol Ther 2003; 18: 231-5.

[7] Ramirez-Cervantes KL, Remes Troche JM, Del Pilar MilkeGarcia M, Romero V, Uscanga LF. Characteristics and factors related to quality of life in Mexican Mestizo patients with celiac disease. BMC Gastroenterol 2015; 15:4.

[8] Rostami K, Malekzadeh R, Shahbazkhani B, Akbari MR, Catassi C. Coeliac disease in Middle Eastern countries: a challenge for the evolutionary history of this complex disorder? Dig Liver Dis 2004 36:694-7.

[9] Corazza GR, Brusco G, Andreani ML, Biagi F, Stefano MD, Gasbarrini G. Previous misdiagnosis and diagnostic delay in adult celiac sprue. J clin Gastroenterol 1996; 22: 324-5.

[10] Sanders DS, Carter MJ, Hurlstone DP, Pearce A, Ward AM, Mc Alindon ME, Lobo AJ. An association of adult celiac disease with irritable bowel syndrome: a case-control study in patients' fulfilling ROME II criteria referred to secondary care. Lancet 2001; 358: 1504-8.

[11] Locke GR III, Murray JA, Zinsmeister AR, Melton LJ III, Talley NJ. Celiac disease serology in irritable bowel syndrome and dyspepsia: a population-based case-control study. Mayo Clin Proc 2004; 79: 476-82.

[12] Agreus L, Svardsudd K, Tibblin G, Lavro B. Endomysium antibodies are superior to gliadin antibodies in screening for coeliac disease in patients presenting supposed functional gastrointestinal symptoms. Scand J Prim Health Care 2000; 18: 105-10.

[13] Sanders DS, Patel D, Stephenson TJ, Ward AM, McCloskey EV, Hadjivassiliou M, Lobo AJ. A primary care cross-sectional study of undiagnosed coeliac disease. Eur J Gastroenterol Hepatol 2003; 15: 407-13.

[14] Chey WD, Nojkov B, Saad RJ, Rai J, Kim CH, Lee DH, Andrews AH, Cash BD. Screening for celiac sprue in patients with suspected irritable bowel syndrome: results from a prospective US multi-center trial. Gastroenterology 2007; 132 (Suppl. 1): A147.

[15] Ozdil K, Sokmen M, Ersoy O, Demirsoy H, Kesici B, Karaca C, Akbayir N, Erdem L et al. Association of gluten enteropathy and irritable bowel syndrome in adult Turkish population. Dig Dis Sci 2008; 53: 1852-5.

[16] Ford AC, Chey WD, Talley NJ, Malhotra A, Spiegel B. Utility of diagnostic tests for celiac diseases in irritable bowel syndrome: systematic review and meta-analysis. Am J Gastro 2008 (Suppl. 1): S 463.

[17] Ford AC, Chey WD, Talley NJ, Malhotra A, Spiegel BM, Moayyedi P. Yield of diagnostic tests for celiac disease in individuals with symptoms suggestive of irritable bowel syndrome: systematic review and meta-analysis. Arch Int Med 2009; 169: 651-8.

[18] Spiegel BMR, De Rosa VP, Gralnek IM, Wang V, Dulai GS Testing for celiac sprue in irritable bowel syndrome with predominant diarrhea: a cost effectiveness analysis. Gastroenterology 2004; 126: 1721-32. 
[19] Rubio-Tapia A, Hill ID, Kelly CP, Calderwood AH, Murray JA American College of Gastroenterology clinical guideline: diagnosis and management of celiac disease. Am J Gastroenterol 2013; 108: 656-77.

[20] Emami MH1, Kouhestani S2, Gholamrezaei A2, Hashemi M2, Mahzouni P3, Raeisi M2, Daghaghzadeh H4, Daneshgar H5: Prevalence of Celiac Disease in Patients with Irritable Bowel Syndrome. Govaresh $\backslash$ Vol. 13\No. 3\ Autumn 2008.

[21] Richard J. Farrell and Ciarán P. Kelly: Celiac disease and refractory celiac disease. 9 th $_{\text {th }}$ edition. Sleisenger and Fordtran's gastrointestinal and liver disease.

[22] Pironti A, Tadeu V, Pedroni A, Porcu A, Manca A, Massarelli G, Realdi G, Dore MP. Role of routine small intestinal biopsy in adult patient with irritable bowel syndrome-like symptoms. Minerva Med. 2010 Jun; 101(3):129-34.

[23] Freeman HJ. Detection of adult celiac disease with duodenal screening biopsies over a 30-year period.Canadian J Gastroenterol. Jul 2013; 27(7): 405-408.

[24] Cash BD, Rubenstein JH, Young PE, Gentry A, Nojkov B, Lee D, Andrews AH, Dobhan R et al. The prevalence of celiac disease among patients with non-constipated irritable bowel syndrome is similar to controls. Gastroenterology 2011; 141: 1187-93.

[25] Choung RS, Rubio-Tapia A, Lahr BD, Kyle RA, Camilleri MJ, Locke GR 3rd, Talley NJ, Murray JA. Evidence against routine testing of patients with functional gastrointestinal disorders for celiac disease: a population based study. Clin Gastroenterol Hepatol 2015; S1542-3565: 00690-4.

[26] Makharia GK, Verma AK, Amarchand R, Bhatnagar S, Das P, Goswami A, Bhatia V, Ahuja V, Datta Gupta S, Anand K. Prevalence of celiac disease in the northern part of India: a community based study. J Gastroenterol Hepatol. 2011 May; 26(5):894-900

[27] Wahnschaffe U, Ullrich R, Riecken EO, Schulzke JD. Celiac disease-like abnormalities in a subgroup of patients with irritable bowel syndrome. Gastroenterology 2001; 121: 1329-38.

[28] De Marchi B, Astegiano M, Sapone N, Bresso F, Pagano N, Bertolusso L, Ponti V, Bruno M et al. Prevalence of coeliac disease in IBS patients in Turin [abstract]. Gastroenterology 2002; 122 (Suppl. 4): A193.

[29] Funka K, Leja M, Bandere B, Gavars D. Low seroprevalence of celiac disease among patients with irritable bowel syndrome in Latvia [abstract]. Gut 2004; 53 (Suppl. VI):A198.

[30] Sood, A., et al., Prevalence of celiac disease among school children in Punjab, North India. Journal of gastroenterology and hepatology, 2006. 21(10): p. 1622-1625.
[31] Yachha, S.K., Celiac disease: India on the global map. Journal of gastroenterology and hepatology, 2006. 21(10): p. 1511-1513.

[32] Kaur, G., et al., Pediatric celiac disease in India is associated with multiple DR3-DQ2 haplotypes. Human immunology, 2002. 63(8): p. $677-682$.

[33] Agrawal, S., et al., Association of human leucocyte-DR and DQ antigens in coeliac disease: A family study. Journal of gastroenterology and hepatology, 2000. 15(7): p. 771-774.

[34] Rani, R., M. Fernandez-Viaea, and P. Stastny, Associations between HLA class II alleles in a North Indian population. Tissue Antigens, 1998. 52(1): p. 37-43.

[35] Ravi kumar, M., et al., Associations of HLA-DRB1, DQB1 and DPB1 alleles with pulmonary tuberculosis in south India. Tubercle and Lung Disease, 1999. 79(5): p. 309-317.

[36] Rostami K, Kerckhaert J, Tiemessen R, von Blomberg BM, Meijer JW, Mulder CJ. Sensitivity of antiendomysium and antigliadin antibodies in untreated celiac disease: disappointing in clinical practice. Am J Gastroenterol. 1999; 94:888-894.

[37] Sanchez-Vargas LA, Thomas-Dupont P, Torres-Aguilera M, Azamar-Jacome AA, et al. Prevalence of celiac disease and related antibodies in patients diagnosed with irritable bowel syndrome according to the Rome III criteria. A case-control study. Neurogastroenterol Motil. 2016 Feb 23.

[38] Khaled Ali Jadallah, Yousef Saleh Khader. Celiac disease in patients with presumed irritable bowel syndrome: A case-finding study. World J Gastroenterol. 2009 November 14; 15(42): 5321-5325.

[39] Patel P, Bercik P, Morgan DG, Bolino C, Pintos-Sanchez MI, Moayyedi P, Ford AC. Prevalence of organic disease at colonoscopy in patients with symptoms compatible with irritable bowel syndrome: cross-sectional survey. Scand J Gastroenterol 2015; 50: 816-23.

[40] Bakhshipour A, Nezam SK, Zakeri Z, Gharibi R, Bahari A, Kaykhaei MA. Coeliac disease in irritable bowel syndrome (Rome III) in Southeast Iran. Arab J Gastroenterol. 2012; 13: 24-27.

[41] Verdu EF, Armstrong D, Murray JA. Between celiac disease and irritable bowel syndrome: the "no man's land" of gluten sensitivity. Am J Gastroenterol 2009; 104: 1587-94.

[42] Aziz I, Lewis NR, Hadjivassiliou M, Winfield SN, Rugg N, Kelsall A, Newrick L, Sanders DS. A UK study assessing the population prevalence of self-reported gluten sensitivity and referral characteristics to secondary care. Eur J Gastroenterol Hepatol 2014; 26: 33-39. 